\title{
THE NATURE OF THE SCATTERING AND FARADAY SCREENS TOWARD THE GALACTIC CENTER
}

\author{
F. YUSEF-ZADEH, P. PARASTARAN \\ Northwestern University \\ Department of Physics and Astronomy \\ Evanston, Il. 60208 \\ M. WARDLE \\ University of Rochester \\ Department of Physics and Astronomy \\ Rochester, N.Y. 14627
}

AND

K. UCHIDA

Max-Planck-Institute fur Radioastronomy

Bonn, Germany

\begin{abstract}
We probe the relationship between the scattering and Faraday screens toward the Galactic center by examining the scattering properties of Sgr $\mathrm{A}^{*}$ and $\mathrm{OH} / \mathrm{IR}$ stars and by measuring the rotation measure distribution of an extended nonthermal filament. The unusually large rotation and scattering measures, the evidence for anisotropic scattering, and the anisotropy in the rotation measure structure function, suggest that the Faraday and scattering media are located near the Galactic center. The results presented here are consistent with a turbulent medium of magnetized thermal electrons with the outer scale of electron density and magnetic field fluctuations being of the order of $5 \times 10^{-4}$ and $2 \mathrm{pcs}$, respectively.
\end{abstract}

\section{Introduction}

The apparent size of the bright compact radio source, $\mathrm{Sgr} \mathrm{A}^{*}$, as a function of wavelength has been extensively studied at a number of frequencies using VLBI measurements (Lo 1993). These high-frequency measure- 
ments indicate that the EW diameter of the source scales as $\lambda^{2.0}$ suggesting that interstellar diffractive scattering is responsible for the broadening. The interstellar scattering hypothesis has received strong confirmation by the measurement of a similar amount of scattering for nearby $\mathrm{OH}$ masers (van Langevelde et al. 1992). Recent VLBA measurements at $\lambda 7 \mathrm{~mm}$ have shown that scattering still dominates the brightness distribution at short wavelengths (Backer 1994).

Recent VLA observation of $\mathrm{Sgr} \mathrm{A}^{*}$ at $\lambda 20 \mathrm{~cm}$ show that the NS diameter has the same wavelength dependence as in the EW direction (Yusef-Zadeh et al. 1994). The scatter-broadened image is elongated in the EW direction, with an axial ratio of $0.56 \pm 0.22$ and a position angle of $82^{\circ} \pm 1.8^{0}$. The conclusion is that there is a 2:1 asymmetry in the scatter-broadened image of $\mathrm{Sgr} \mathrm{A}^{*}$ at a number of wavelengths with a position angle that ranges between 80 to $90^{\circ}$. Elongation of the scattered image with nearly constant PA and ellipticity is explained by propagation through anisotropic turbulent distribution of thermal electrons (Higdon 1984). Similar effects have been observed in radio wavelengths through the outer solar corona where the magnetic field dominance over plasma pressure leads to highly anisotropic turbulence (Narayan et al. 1989; Armstrong et al. 1990).

Recent observations show that the scattering of the $\mathrm{OH}$ masers within the inner $45^{\prime}$ of the Galactic center is also anisotropic (Frail et al. 1994). Yusef-Zadeh et al. argue that the scattering occurs within HII regions lying in the central $100 \mathrm{pc}$ of the Galaxy. The magnetic field in this region must be organized and have a strength of at least a milliGauss. Such a high value of the magnetic field is not unexpected since a number of large-scale magnetized filaments in the Galactic center region are thought to have milliGauss field strengths in the direction perpendicular to the Galactic plane. The inferred magnetic field from the scatter-broadened sources indicate a strong magnetic field with a random orientation within the inner $45^{\prime}$ and therefore, they do not follow the organized poloidal geometry of the field which is expected to dominate the Galactic center region (Yusef-Zadeh 1989; Morris 1994). The anisotropic scattering results complement polarization and Zeeman measurements in characterizing the magnetic field in a turbulent medium of thermal electrons.

\section{The Scattering Medium}

The anisotropy of $\mathrm{OH} / \mathrm{IR}$ stars and Sgr $\mathrm{A}^{*}$ is probably caused by a magnetic field permeating the scattering medium, but the location of the scattering screen has not been determined. The strong, anisotropic scattering extending over a $40^{\prime}$ region aligned with the Galactic center is suggestive of a scattering medium near the Galactic center. However, scattering be- 
comes less effective when the distance from the source to the screen is much smaller than their distance to the observer because the broadening must overcome the spherical divergence of the wavefront incident on the screen. If the scattering medium is located near the Galactic center the electron density fluctuations (for an outer turbulent scale of $1 \mathrm{pc}$ ) would produce more free-free emission than is observed from the galactic center and in addition, the screen would become optically thick to low radio frequencies (Backer 1978). This argues for the coincidental alignment of a screen that lies outside the central regions of the Galaxy (e.g. van Langenvelde et al 1992)

An important point is that the angular broadening reflects the power in the screen at the smallest length scales (perhaps $10^{7} \mathrm{~cm}$ ), whereas the size of the density fluctuations depends on the power on the largest scales. Estimates of the electron density fluctuations are obtained from the observations by extrapolating the power spectrum of the fluctuations from $10^{7}$ $\mathrm{cm}$ to an unknown outer scale, typically assumed to be of order $10^{18} \mathrm{~cm}$ or more (a typical HII region size). It is possible for the scattering screen to lie within a few tens of parsecs of the Galactic center if the outer scale is much smaller than $1 \mathrm{pc}$. One possibility is that the scattering occurs in thin ionized surface layers of molecular clouds lying in the central $100 \mathrm{pc}$ of the Galaxy (Yusef-Zadeh et al. 1994). The outer scale of turbulence in this model is identified with the thickness of the ionized layers, about $4 \times 10^{-4} \mathrm{pc}$ for the intense UV radiation field in the region.

The assumptions made in the above model are that the UV radiation field is strong enough to photoionize the outer layer of the molecular clouds and that the outer scale of turbulence is identified with the thickness of the photoionized layer. Thus, measuring the radiation field will test this scenario. One method of investigating the nature of the UV radiation field in the inner Galaxy is to search for radio continuum emission from the outer envelopes of the population of $\mathrm{OH} / \mathrm{IR}$ stars that are known to lie in the Galactic center region (Lindqvist et al. 1992). The ionized surfaces of the stellar envelopes should emit an amount of free-free emission dependent on the value of the ambient UV flux near the Galactic center. The strength of the free-free emission can be compared to theoretical estimates of the fluxes from photoionized surfaces of mass-losing stellar envelopes (Serabyn et al. 1991). The continuum fluxes from these cool stars will reflect the variation in the local UV flux with distance from the Galactic center.

\section{The Faraday Screen}

It is plausible that the scattering screen and Faraday screens should be identified with one another. In order to examine the relationship between 
these two screens, we analyzed polarization data of a nonthermal radio continuum filament, G359.5+0.18 (Bally and Yusef-Zadeh 1989). This source is located within the region where strong anisotropic scattering of thermal emission from $\mathrm{OH} / \mathrm{IR}$ stars has been found, and allows us to measure the variation of Galactic Faraday rotation over small angular scales along its $3^{\prime}$ length.

Multifrequency VLA observations reveal the magnetic field geometry, and detailed rotation measure distributions of this system of nonthermal filaments with a spatial resolution of $\approx 3$ ". The projected magnetic field follows predominantly along the filaments except in its western boundary where the intrinsic field runs perpendicular to the direction of the linear filaments. In addition, the filaments show very high rotation measures (RM) ranging between -370 and $-4200 \mathrm{rad} \mathrm{m}^{-2}$. The plots of the RM values vs. $\lambda^{2}$ demonstrate that the Faraday screen is external to the source (Yusef-Zadeh et al. 1995).

The high variability of the rotation measure along G359.5+0.18 is a powerful probe of the structure of the ionized magnetized medium that constitutes the Faraday screen (and probably the scattering screen). The variation in RM over an angular scale $(\delta \theta)$ is described by the structure function, which for a Kolmogorov spectrum increases as $\delta \theta^{5 / 3}$ for small angular scales and flattens at scales larger than the outer turbulent scale (Simonetti and Cordes 1988; Clegg et al. 1992). The observed structure function has a power-law index of approximately 2 for small scales and flattens above 10". The RM structure function distribution indicates that for $\delta \theta<20$ ", the RM gradient along the Galactic plane appears to be lower by a factor 2 than in other directions.

This anisotropy is roughly consistent with the anisotropic scattering seen toward $\mathrm{Sgr} \mathrm{A}^{*}$ and the Galactic center $\mathrm{OH} / \mathrm{IR}$ stars, suggesting that the same magnetized medium is responsible for the large anisotropic scattering and the variation of the RM on small angular scales. Further evidence is provided by the high RM found for a number of other sources located within a degree from the Galactic center (Inoue et al. 1984; Yusef-Zadeh and Morris 1987; Gray 1994).

Both the magnitude of the RM and its small scale structure argue against the ionized screen being an extended local HII region coincidentally aligned with the Galactic center. The RM toward G359.5+0.18 is more than two orders of magnitude higher than other sources outside the Galactic center region, requiring $n_{e} B_{\|} L \approx 3000 \mathrm{~cm}^{-3} \mu \mathrm{G} \mathrm{pc}$, where $L$ is the thickness of the screen. For magnetic fields in the screen of a few microGauss, the emission measure $n_{e}^{2} L$ exceeds the value $2 \times 10^{4} \mathrm{~cm}^{-6} \mathrm{pc}$ seen towards the galactic center (Mezger and Pauls 1979) unless $L$ is in excess of $50 \mathrm{pc}$. Further, the RM fluctuates wildy on angular scales of a few arc- 


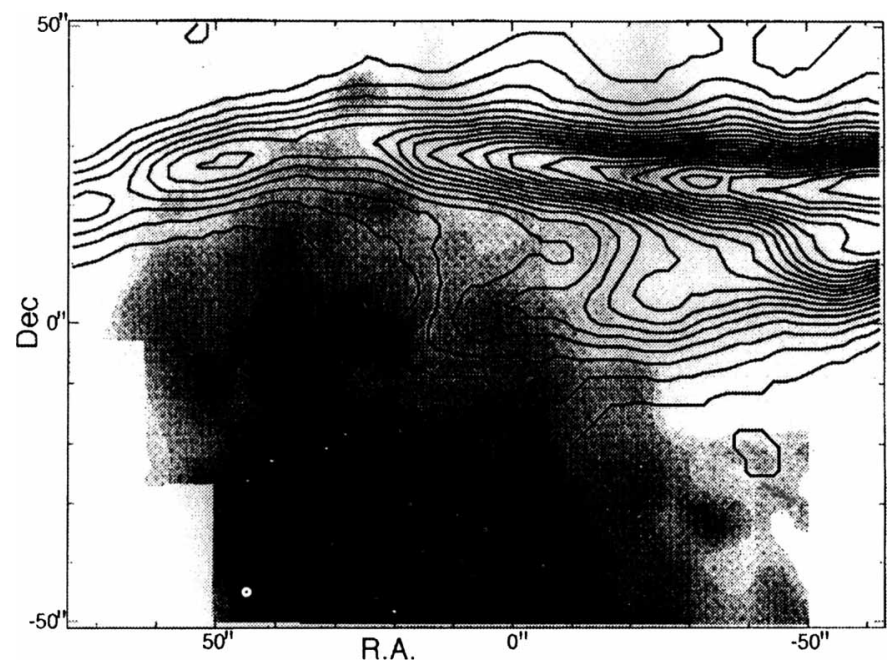

Figure 1. Contours of the $\lambda 6 \mathrm{~cm}$ continuum image superimposed on a grayscale $\mathrm{J}=2-1$ ${ }^{12} \mathrm{CO}$ line emission integrated from -160 to $-122 \mathrm{~km} \mathrm{~s}^{-1}$ based on IRAM observations. It is slightly to the east of this region where the linear filaments, as seen in Figure 2, lose their coherent appearance.

seconds. $L$ cannot be much greater than the corresponding spatial scale otherwise averaging along the line of sight would substantially reduce the amplitude of the fluctuations.

In summary, the RM observations strongly suggest that the Faraday screen is located near the Galactic center, where the electron density and magnetic field fluctuations are expected to have large values relative to the rest of the Galaxy. If the Faraday screen is to be identified with the photoionized surfaces of clouds in the region, the 10" angular scale must represent the outer scale of the fluctuations in the magnetic field (about $0.5 \mathrm{pc}$ ) rather than the electron density fluctuations.

\section{G359.5+0.18 and Its Adjacent Molecular Cloud}

A number of molecular clouds lying within the inner few hundred pcs of the Galaxy appear to be outlined by nonthermal filaments. The interaction hypothesis between the molecular gas and the energetic electrons associated with synchrotron-emitting filaments has been discussed (e.g., Serabyn 1994), though the evidence for such an interaction is still not convincing. As a point of illustration, we show $J=2-1 \mathrm{CO}$ observations of the molecular cloud that lies at the edge of G359.54+0.18. Earlier $\mathrm{J}=2-1 \mathrm{CS}$ and $\mathrm{J}=1-0$ 


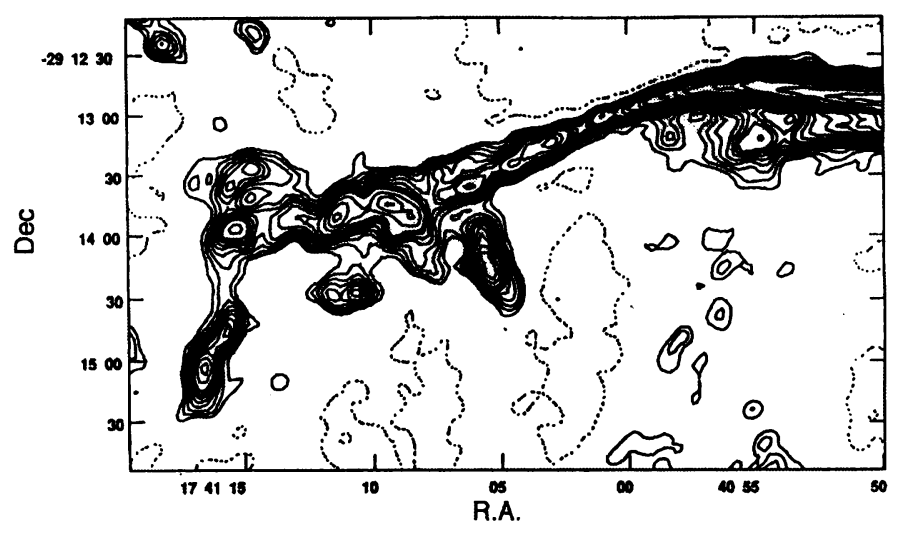

Figure 2. The $\lambda 6 \mathrm{~cm}$ total intensity images of the eastern region of the filaments with a resolution of $10 " \times 10^{\prime \prime}$ are displayed.

${ }^{13} \mathrm{CO}$ measurements from the Bell Labs survey data with a resolution of about 100" (Bally and Yusef-Zadeh 1989) and J=2-1 ${ }^{12} \mathrm{CO}$ measurements based on observations made with the NRAO $12 \mathrm{~m}$ telescope with a resolution of 30" (Bally, Yusef-Zadeh and Hollis 1989) suggested the possible association between the filaments in G359.54+0.18 and the adjacent -140 $\mathrm{km} \mathrm{s}^{-1}$ molecular cloud. Motivated by the results of earlier low-resolution measurements, $\mathrm{J}=2-1{ }^{12} \mathrm{CO}$ observations were carried out toward the filament G359.54+0.18 with a resolution of 12 " using the IRAM $30 \mathrm{~m}$ telescope. The new CO map which is superimposed on the radio continuum image of G359.5+0.18 is shown in Figure 1. There is an apparent anti-correlation of the continuum nonthermal filaments with the molecular cloud suggesting that these two features maybe physically interacting with each other. Figure 2 shows contours of continuum emission at $\lambda 6 \mathrm{~cm}$ from a region slightly east of the area that $\mathrm{CO}$ distribution was shown in Figure 1. The distorted filaments lose their coherent appearance as their morphology strengthens the argument for the interaction of G359.5+0.18 with its adjacent cloud. A more detailed presentation of these observations will be given elsewhere.

\section{References}

Armstrong, J., et al. 1990, $A p J, 358,685$.

Backer, D.C. 1994, this conference 
Bally, J., Yusef-Zadeh, F. 1989, ApJ, 336, 173.

Clegg, A.W., Cordes, J.M., Simonetti, J.H. and Kulkarni, S.R. 1992, ApJ, 386, 143.

Frail, D.A., Diamond, P.J., Cordes, J.M. and van Langevelde, H.J. 1994, ApJ, 427, L43. Gray, A.D. 1994, PhD. Thesis, University of Sydney.

Higdon, J.C. $1993, A p J, 285,109$.

Inoue, M. et al. 1984, Pub.Astron.Soc.Japan., 36, 633.

Lindqvist, M., Habing, H.J. and Winnberg, A. 1992, A.A., 259118.

Lo et al. 1993, Nature, 362, 38.

Morris, M. 1994, this conference.

Narayan, R., et al. 1989, MNRAS, 241, 403.

Mezger, P.G., and Pauls, T. 1985, in IAU Symposium No. 84, ed. W.B. Burton, Reidel, Dordrecht, p. 357.

Morris, M. 1994, this conference.

Serabyn, E., Lacy, J.H., and Achtermann, J.M. 1991, ApJ, 378, 557.

Simonetti, J.H. and Cordes, J.M. 1988, in AIP 174, Radio Waves Scattering in the Interstellar Medium, ed. B.J. Ricket and D.C. Backer (NY:AIP)

van Langevelde, H.J., Frail, D.A., Cordes, J.M. and Diamond, P.J. 1992, ApJ, 396, 686.

Yusef-Zadeh, F., 1989, IAU Symposium No. 136, The Center of the Galaxy, ed. M. Morris, Kluwer, Dordrecht, p. 243.

Yusef-Zadeh, F. et al. 1995, in preparation.

Yusef-Zadeh, F. and Morris, M. 1987, ApJ, 322, 721. 